Ciência Florestal, Santa Maria, v. 20, n. 3, p. 411-418 jul.-set., 2010

ISSN 0103-9954

\title{
ESTRUCTURA POBLACIONAL DE Myrocarpus frondosus Allemão EN UN BOSQUE EN GALERÍA DE LA SELVA PARANAENSE EN MISIONES, ARGENTINA
}

\author{
POPULATION STRUCTURE OF Myrocarpus frondosus Allemão IN A RIPARIAN AREA OF \\ THE PARANAENSE FOREST IN MISIONES, ARGENTINA
}

Silvana Lucia Caldato ${ }^{1}$ Raúl Vicente Pezzutti ${ }^{1}$

\begin{abstract}
RESUMEN
Para describir la estructura poblacional de Myrocarpus frondosus Allemão fue estudiada una población de la especie, caracterizando los estadios de tamaño y la distribución espacial de los individuos, en un bosque

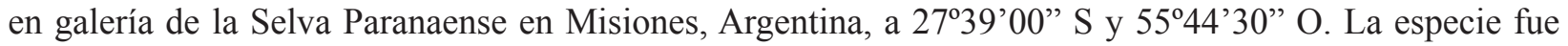
estudiada en parcelas circulares. En las parcelas fueron considerados todos los individuos de Myrocarpus frondosus con diámetro DAP (a 1,3 metros de altura) mayor o igual a $10 \mathrm{~cm}$ para el estrato arbóreo y para la regeneración natural fueron considerados los plantines de la especie con DAP variando de $2 \mathrm{~cm}$ hasta 9,9 $\mathrm{cm}$. Fueron encontrados 396 individuos (431 ind. ha ${ }^{-1}$ ), de los cuales 180 pertenecieron al estrato arbóreo y 216 correspondieron a la regeneración natural. Los resultados muestran que la amplitud de tamaño en diámetro varió desde $2 \mathrm{~cm}$ hasta $50 \mathrm{~cm}$, con una distribución de la población estudiada en forma de J invertida, representando una población con substancial reclutamiento de individuos en sus primeras etapas de desarrollo. La especie presentó un patrón agrupado de distribución espacial.
\end{abstract}

Palabras-clave: estructura de tamaños; distribución espacial; conservación de especies.

\begin{abstract}
In order to describe the population structure of Myrocarpus frondosus Allemão, the structure of size class and the spatial distribution of the individuals species was studied, in a riparian area of the Paranaense Forest in Misiones, Argentina, at $27^{\circ} 39^{\prime} 00^{\prime \prime} \mathrm{S}$ and $55^{\circ} 44^{\prime} 30^{\prime \prime} \mathrm{W}$. The species was studied in circular plots. All the individuals of Myrocarpus frondosus with diameter greater or equal to $10 \mathrm{~cm}$ (at $1.3 \mathrm{~m}$ height - DBH) were considered for the tree stage, and all seedlings with diameter ranging from $2 \mathrm{~cm}$ to $9.9 \mathrm{~cm}$ were considered for natural regeneration. Three hundred and ninety six individuals were found (431 ind. ha ${ }^{-1}$ ), with 180 individuals in the tree stage and 216 individuals in the natural regeneration stage. The results show that the diameter size of the population varied from $2 \mathrm{~cm}$ to $50 \mathrm{~cm}$ with reverse $\mathrm{J}$ distribution, representing a substantial recruitment in the younger age classes. The species presented a clustered pattern of spatial distribution.
\end{abstract}

Keywords: size structure; spatial distribution; species conservation.

\section{INTRODUCCION}

El incienso (Myrocarpus frondosus Allemão), perteneciente a la familia Fabaceae, está fuertemente amenazado por pérdida de hábitat. La especie se incluye en la lista de especies argentinas en peligro o vulnerables (CHEBEZ, 1994). En la Selva Misionera el incienso constituye, junto al lapacho (Handroanthus heptaphyllus (Vell.) Mattos), el cedro (Cedrela fissilis Vell.), el peteribí (Cordia trichotoma (Vell.) Arráb. ex Steud.) y la araucaria (Araucaria angustifolia (Bertol.) Kuntze), una de las especies arbóreas proveedoras de las maderas más preciadas, siendo representantes valiosísimos de la flora nativa. El incienso pertenece al estrato arbóreo superior, alcanzando alturas de hasta $25 \mathrm{~m}$ y troncos de $1 \mathrm{~m}$ de diámetro, con largo fuste recto y copa poco extendida. Especie propia de Brasil en la selva Estacional Decidual (REITZ et al., 1988), Paraguay y nordeste de Argentina (DIMITRI,

1. Engenheiro Florestal, MSc., Doutorando do Programa de Pós-graduação em Engenharia Florestal, Centro de Ciências Rurais, Universidade Federal de Santa Maria, Av. Roraima, 1000, CEP 97105-900, Santa Maria (RS). Bolsista do CNPq.scaldato@yahoo.com.br/raulpezzutti@yahoo.com.br Recebido para publicação em 14/01/2009 e aceito em 05/04/2010. 
2000). Según el autor su nombre común tiene origen en los tiempos de las antiguas misiones jesuíticas que habitaron el nordeste argentino. En estas se lo utilizaba por sus características balsámicas, ya que efectuando incisiones en la corteza exuda una resina aromática semejante a la mirra, que se utilizaba en los templos en reemplazo del verdadero incienso.

La Selva Paranaense representa la formación vegetal natural de la provincia de Misiones, cuya vegetación está compuesta por un gran número de especies arbóreas en diferentes estratos. El desarrollo que alcanza esta selva decrece desde el norte hacia el sur, y en su región meridional se encuentra una zona de transición, con vegetación de pastos asociados a bosques de galerías, que luego deja lugar a la zona de campos (MARTINEZ CROVETTO, 1963).

Estudios sobre la dinámica, regeneración y demografía son considerados esenciales para el entendimiento de los procesos que regulan la dinámicay la estructura de las comunidades forestales (BROKAW, 1985; FORGET, 1992; ARISTA, 1995). El manejo de los bosques para explotación o conservación depende del entendimiento de la dinámica poblacional de las plantas dominantes (VAN GROENENDAEL et al., 1996). Según Viana et al. (1992) los fragmentos forestales tienden a asumir una importancia creciente en la conservación de la diversidad biológica de los bosques. Debido a la acción antropogénica con el uso intensivo del suelo las áreas de conservación representan un papel fundamental en este sentido.

El presente trabajo tiene por objetivo analizar la estructura poblacional de Myrocarpus frondosus en un área de bosque en galería de la Selva Paranaense en Misiones, Argentina.

\section{MATERIALES Y METODOS}

\section{Área de estudio}

El presente trabajo fue desarrollado en el bosque nativo en galería del predio San Andrés perteneciente a la empresa Forestal Bosques del Plata S.A., con un área protegida de bosques de 300 ha, ubicado en la Provincia de Misiones, Argentina, entre las coordenadas geográficas de $27^{\circ} 39^{\prime} 00^{\prime \prime}$ de latitud Sur y $55^{\circ} 44^{\prime} 30^{\prime \prime}$ de longitud Oeste de Greenwich; con una altitud media de $120 \mathrm{~m}$ sobre el nivel del mar. El área de bosque nativo del predio limita con el Parque Provincial Fachinal y con áreas rurales. El Parque Fachinal (Ley P. No 3358 ) de 51 ha, se encuentra localizado sobre lo alto de un cerro con vertientes temporarias que desaguan en la cuenca del Arroyo Garupá el cual desemboca en el Río Paraná.

El área la cual se realiza el estudio presenta un interesante mosaico de vegetación. La vegetación boscosa corresponde al Distrito de las Selvas Mixtas, denominada Selva Paranaense (CABRERA, 1994). El bosque misionero, ubicado en el extremo noreste del predio, es una valiosa muestra del distrito misionero del urunday (Myracrodruon balansae (Engl.) Santin) y representa el límite austral de la Selva Paranaense.

La Selva Misionera o Paranaense, tiene todas las características de las selvas lluviosas cálidas del mundo: bioma típico de zonas de elevada temperatura y humedad y sin fluctuaciones marcadas y sin una estación seca definida. Se caracteriza por su masa boscosa de 20 a $30 \mathrm{~m}$ de altura integrada por gran variedad de especies vegetales (GARELLO, 1998). El clima de la zona es Cfa (KÖPPEN, 1948), siendo un clima mesotermal, cálido templado, sin estación seca, con precipitación máxima en el otoño y veranos muy cálidos. La temperatura media anual es de $20,5^{\circ} \mathrm{C}$ y la precipitación media anual de 1800 $\mathrm{mm}$.

Los suelos de la región son formaciones de basalto, en unos ambientes de transición entre pajonales mesófilos, comunidades pioneras y selva con urunday y selva mixta. El relieve representa las últimas estribaciones de las sierras del Imán en Misiones y sus planicies de erosión, la unidad de relieve ondulado está integrado por lomadas redondeadas de tipo cupuliforme, su rasgo más saliente es la existencia de superficies de erosión de antiguos pediplanos y pedimentos que dan al paisaje el aspecto de suaves colinas escalonadas (CARNEVALI, 1994).

La hidrología del área tiene sus vertientes temporarias que desaguan en la cuenca del Arroyo Garupá, lo cual tiene un área de aporte de $730 \mathrm{~km}^{2}$, su principal afluente es el arroyo Pindapoy Grande, a su vez este recibe aporte de otros como arroyo Guazupí, con varios tributarios que escurren en el área del predio San Andrés, cuyas nacientes se encuentran en las ondulaciones de las denominadas Sierras del Imán (IGM, 1938; EBY, 1998).

\section{Aspectos ecológicos de la especie}

El incienso es una especie secundaria inicial, crece en vegetación secundaria, principalmente en fundo de valles, inicio de pendientes escarpadas y 
en otros lugares con suelos húmedos donde puede tornarse frecuente. Los frutos deben ser colectados cuando cambian de color, para esto se recomienda escalar el árbol y derrumbar las ramas conteniendo los frutos maduros, la maduración de los frutos va de noviembre a febrero. La dispersión de los frutos es anemocórica y la longevidad del almacenamiento de las semillas es corta. Su regeneración en el bosque denso y desarrollado no es muy acentuada; las semillas se desarrollan preferentemente en locales con cierta luminosidad. Es una especie melífera y toda la planta es aromática (REITZ et al., 1988). La madera del incienso tiene cierto contenido de tanino, resinas y gomorresinas que favorecen su utilización a la intemperie (LORENZI, 1998).

\section{Muestreo}

Para estudiar la estructura poblacional y distribución espacial de Myrocarpus frondosus, en Julio de 2006 fueron instaladas 13 parcelas circulares con $30 \mathrm{~m}$ de diámetro $\left(707 \mathrm{~m}^{2}\right)$, totalizando 0,91 ha de área de muestreo en donde se censaron todos los individuos de incienso. La distribución de las parcelas en el bosque en galería fue al azar en el sentido paralelo al arroyo, cubriendo una superficie longitudinal de $5 \mathrm{~km}$. En cada parcela fueron tomadas las coordenadas geográficas en el centro de la parcela con el uso del GPS.

\section{Obtención de los datos}

Para caracterizar el estrato arbóreo fueron identificados y medidos el diámetro a la altura del pecho (DAP) a 1,30 m do solo, de los individuos de incienso con DAP igual o superior a $10 \mathrm{~cm}$, clasificados en clases artificiales de diámetro como sigue: $\mathrm{DAP}=10<20, \mathrm{DAP}=20<30, \mathrm{DAP}=30<40$, $\mathrm{DAP}=40<50 ;$ y para clasificar la regeneración natural considerados los individuos con DAP igual o superior a $2 \mathrm{~cm}$ hasta $9,9 \mathrm{~cm}$, divididos en dos categorías: (i) de $2 \mathrm{~cm}$ hasta $4,9 \mathrm{~cm}$ y categoría (ii) de $5 \mathrm{~cm}$ hasta $9,9 \mathrm{~cm}$. Con la información obtenida se estimó la densidad (individuos ha ${ }^{-1}$ ), distribución de tamaños (frecuencia por clase diamétrica) y distribución espacial. Para analizar el patrón de distribución espacial se consideró el índice de dispersión de Morisita (1959), una cualidad de este índice es que el mismo es poco influenciado por el tamaño de las parcelas y presenta excelentes cualidades para la detección del grado de dispersión de la especie. El índice puede asumir el valor 1,0 en el caso de dispersión al azar, un valor menor a 1,0 para la dispersión uniforme y, mayor a 1,0 para distribución agregada, según la siguiente ecuación:

$I_{M o r}=n \frac{\sum X^{2}-N}{N(N-1)}$

Donde:

$n$ = número de parcelas;

$\sum X^{2}=\sum f(X) X^{2}=$ suma de cuadrados del número de individuos multiplicado por $f(X)$;

$f(X)=$ frecuencia de individuos;

$N=\sum f(X)=$ frecuencia total de individuos encontrados en todas las $\mathrm{n}$ parcelas.

\section{RESULTADOS Y DISCUSION}

\section{Distribución de tamaños}

La densidad total de individuos de Myrocarpus frondosus muestreados fue de 396 individuos (431 ind. ha ${ }^{-1}$ ), de los cuales 216 pertenecieron a la regeneración natural, siendo 54 individuos en la categoría (i) de 2 hasta $4,9 \mathrm{~cm}$ y 162 plantas en la categoría (ii) de 5 hasta $9,9 \mathrm{~cm}$; y 180 como árboles en distintas clases diamétricas. Los individuos estudiados presentaron una amplitud de tamaño en diámetro desde $2 \mathrm{~cm}$ hasta $50 \mathrm{~cm}$. La distribución de la estructura de tamaños se encuentra representada en la Figura 1, donde se puede observar que la especie presenta una estructura de distribución de tamaños en forma de $\mathrm{J}$ invertida; con mayor número de individuos en los centros de clases diamétricas de 7 y $15 \mathrm{~cm}$, representando una población conformada principalmente por individuos jóvenes.

Webb et al. (1972), verificaron que patrones de crecimiento inicial lento resultan en distribución en la forma $J$ invertida, una vez que los individuos permanecen en la misma clase diamétrica por largo período de tiempo. El modelo de distribución en J invertida sugiere que las poblaciones que componen una comunidad son estables y regenerativas. Este tipo de modelo es ideal en situaciones de manejo sustentable de poblaciones arbóreas que ajusta su distribución diamétrica de acuerdo con este modelo.

De acuerdo con Condit (1995) el tamaño de los árboles y la distribución de los individuos son parámetros utilizados para evaluar la salud de una población en un bosque. Para conocer los cambios 
que ocurrieron en el pasado y predecir el futuro, se realizan estudios de la distribución diamétrica de las especies. El número elevado de individuos jóvenes indica que la población está estable y creciente. Sin embargo, el comportamiento de una especie está estrictamente asociado al ambiente en que pueden ocurrir, por lo tanto, si el ambiente varia, la tendencia es variar en diferentes sentidos, en reducción o expansión dependiente de su comportamiento (ODUM, 1972). En el caso de Myrocarpus frondosus la actual distribución de tamaños de los individuos coincide con el estadio sucesional secundario del bosque estudiado, donde hay luz suficiente para que las especies secundarias se puedan desarrollar.

\section{Distribución espacial}

El índice de Morisita para la población de Myrocarpus frondosus estudiada fue de 1,71 lo que representa un patrón de dispersión agregado para la especie, y de acuerdo a Martinez-Ramos (1985) las poblaciones de especies pioneras o secundarias como es el caso del incienso, crecen segregadas en el espacio.
Varios factores pueden influenciar para definir un patrón de distribución agregado. Algunas poblaciones se encuentran agregadas en los sitios donde han ocurrido claros producidos por la caída de árboles, otras se agregan debido a pobres niveles de dispersión de semillas y baja depredación de las mismas, y otras muestran agregación en relación con hábitat topográficos y edáficos específicos (MARTINEZ-RAMOS y ALVAREZ-BUYLLA, 1995). Para el incienso el bosque estudiado con suelos húmedos mostró ser un hábitat muy propicio para su desarrollo.

El conocimiento del patrón de distribución de las especies es fundamental en la toma de decisión sobre el manejo a ser aplicado en el área. De acuerdo con Martins et al. (2003) en estudio sobre el efecto de la explotación selectiva en un bosque estacional semidecidual en Paraná, la mayoría de las especies presentó tendencia a la agregación, seguida por el patrón agregado y, con menor frecuencia por el patrón al azar. La ocurrencia de este patrón es común en bosques tropicales, donde especies abundantes ocurren agrupadas o con tendencia al agrupamiento. Según los autores después de la explotación, la mayoría de las especies presentó un

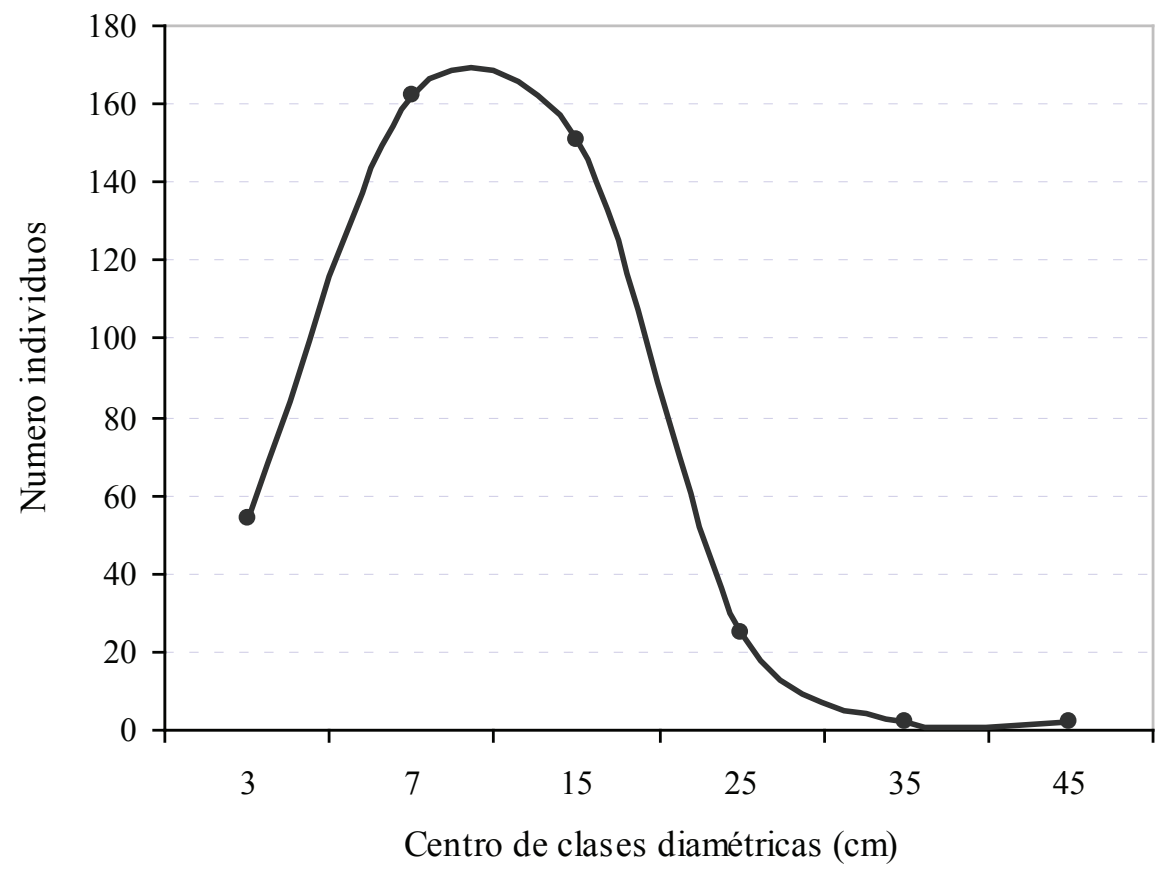

FIGURA 1: Distribución de los individuos de Myrocarpus frondosus en clases de tamaño (centro de clases diamétricas - DAP), en un bosque en galería de la Selva Paranaense en Misiones - Argentina.

FIGURE 1: Distribution of individuals of Myrocarpus frondosus in size classes (diameter center class $\mathrm{DBH})$, in a riparian area of the Paranaense Forest in Misiones, Argentina.

Ci. Fl., v. 20, n. 3, jul.-set., 2010 
patrón de distribución al azar.

La Figura 2 muestra gráficamente la distribución espacial de Myrocarpus frondosus por clase de tamaños en las parcelas circulares de $30 \mathrm{~m}$ de diámetro. En dos parcelas (5 y 13) no se registró la presencia de individuos de incienso. De acuerdo con la Figura 2 de las 11 parcelas con la presencia de la especie 10 parcelas presentaron individuos en la fase de regeneración natural; en 4 parcelas hubo la presencia de 3 clases de tamaño y en 3 parcelas la ocurrencia de 4 clases representando todos los tamaños. Esta variación de parcelas con muchos individuos y de todas las clases y otras sin la presencia de individuos confiere la distribución agrupada de la especie. La presencia de la regeneración natural en casi todas las parcelas indica la adaptación y la colonización de la especie en el área, lo que puede ser justificado por las características del ecosistema
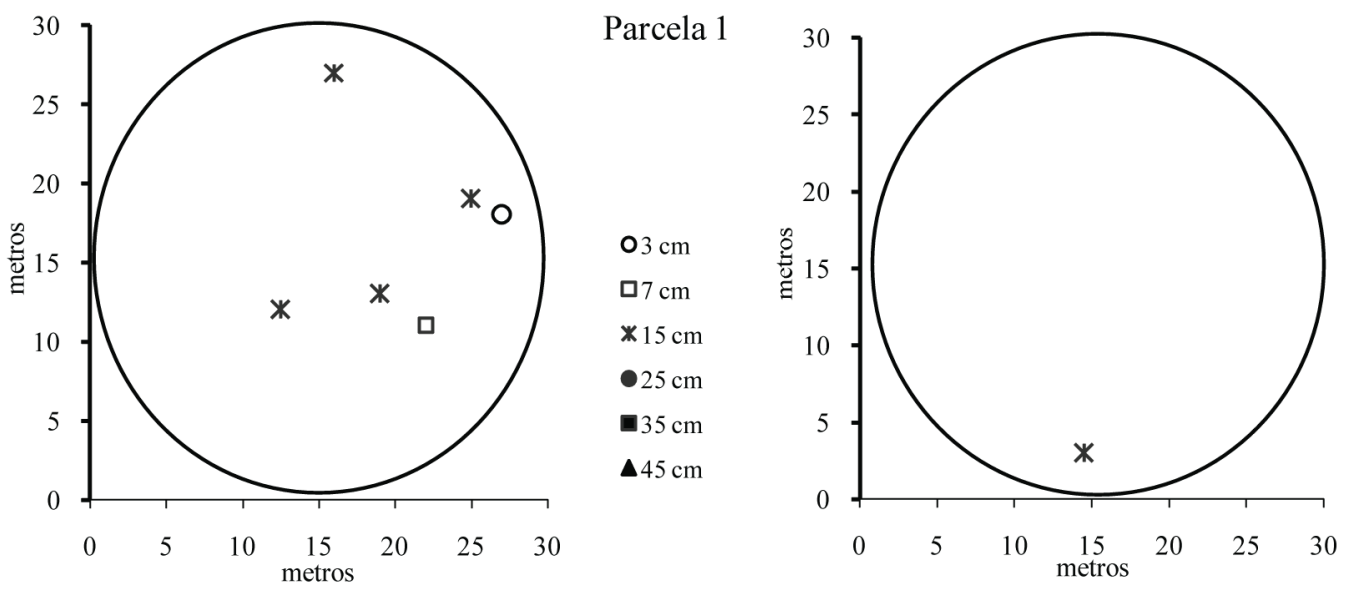

Parcela 2
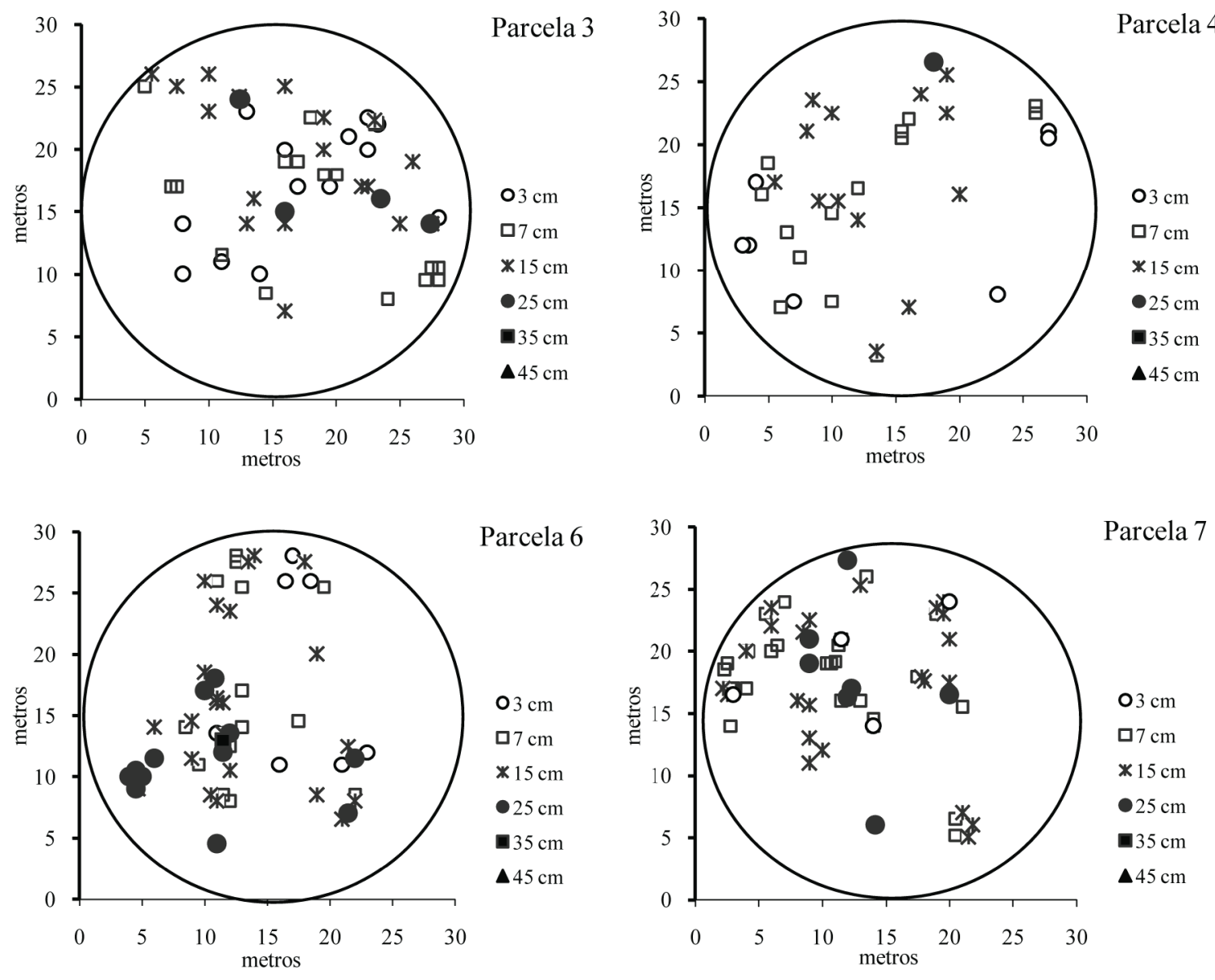

Continua... 

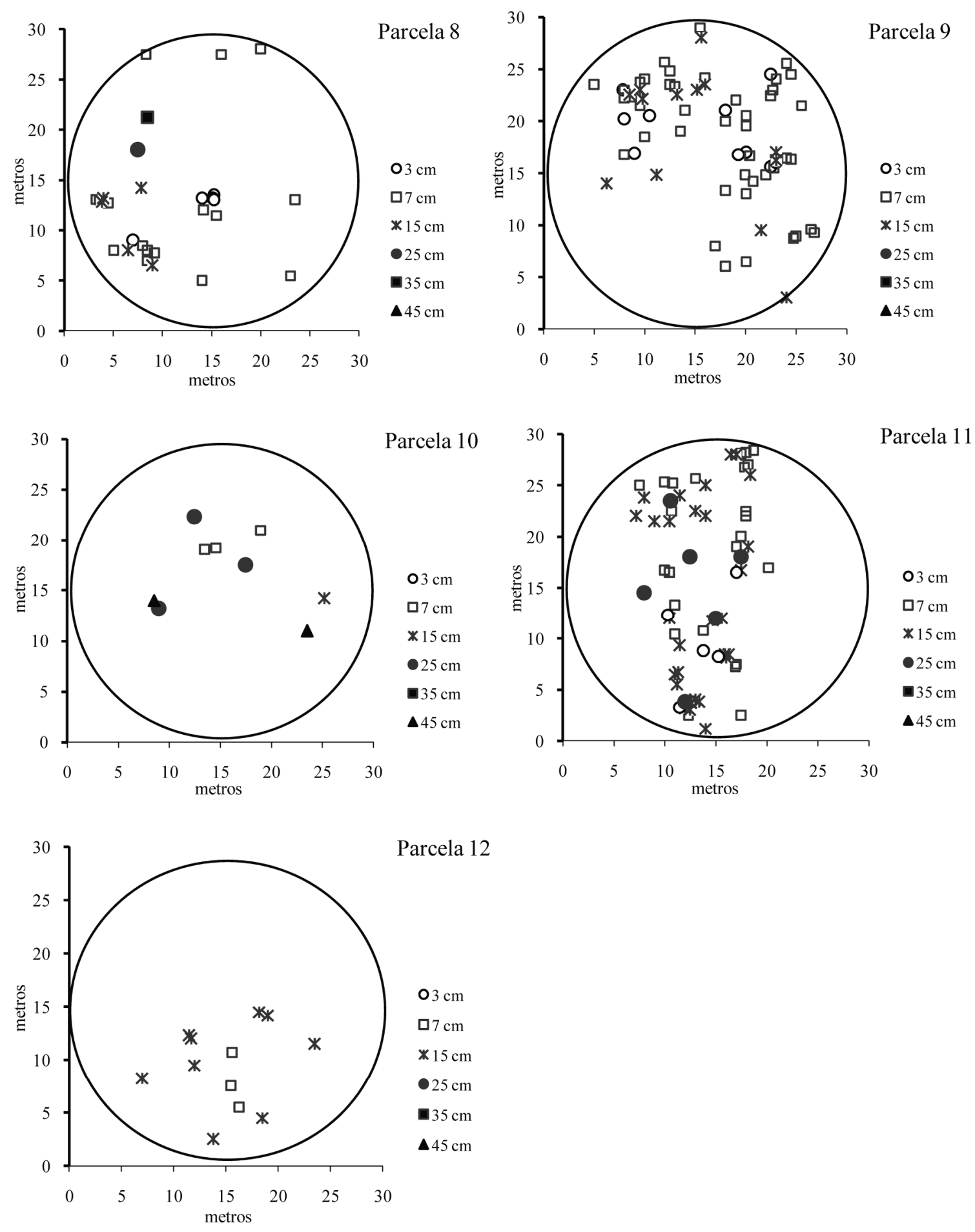

FIGURA 2: Distribución espacial de los individuos de Myrocarpus frondosus por clases de tamaños (centro de clase diamétrica - $\mathrm{cm}$ ) en las parcelas circulares en el bosque en galería de la Selva Paranaense en Misiones, Argentina.

FIGURE 2: Spatial distribution of individuals of Myrocarpus frondosus by size class (diameter center class $-\mathrm{cm}$ ) in circular plots, in a riparian area of the Paranaense Forest in Misiones, Argentina.

Ci. Fl., v. 20, n. 3, jul.-set., 2010 
donde la especie fue estudiada, o sea, bosque en galería (ambiente húmedo) y en fase de sucesión secundaria.

\section{CONCLUSIONES}

La estructura poblacional de Myrocarpus frondosus presenta una forma de distribución de $\mathrm{J}$ invertida, indicando una entrada continua de nuevos individuos en la población estudiada.

La especie presenta un patrón de distribución agregado.

El área estudiada presenta una alta densidad de individuos, con presencia en diversas clases de tamaños, variando desde $2 \mathrm{~cm}$ hasta $50 \mathrm{~cm}$ de diámetro, con lo cual, se podría considerar a todo el bosque en galería como área productora de semillas de incienso en programas de conservación para la especie.

\section{AGRADECIMIENTOS}

Los autores agradecen el apoyo en la colecta de los datos de campo a José Veron y Alfredo Medina, y a la empresa Bosques del Plata por la disponibilidad de los datos para publicación.

\section{REFERENCIAS BIBLIOGRAFICAS}

ARISTA, M. The structure and dynamics of an Abies pinsapo forest in southern Spain. Forest Ecology and Management, Amsterdam, v. 74, n. 1-3, p. 81-89, June 1995.

BROKAW, N. V. L. Treefalls, regrowth, and community structure in tropical forests. In: PICKETT, S.T.A.; WHITE, P.S. (Eds.). The ecology of natural disturbance and patch dynamics. New York: Academic Press, 1985. Cap. 4, p. 31-49.

CABRERA, A. Regiones fitogeográficas Argentinas: enciclopedia argentina de agricultura y jardinería. 2. ed. Buenos Aires: Acme, 1994. 85 p. t.2.

CARNEVALI, R. Fitogeografía de la Provincia de Corrientes. Corrientes: Gobierno de la Provincia de Corrientes e Instituto Nacional de Tecnología Agropecuaria (INTA), 1994. 324 p.

CONDIT, R. Research in large, long-term tropical forest plots. Trends in Ecology and Evolution, Amsterdam, v.10, n.1, p. 18-22, Jan.1995.

CHEBEZ, J. C. Los que se van: especies argentinas en peligro. 1. ed. Buenos Aires: Albatros, 1994. 606 p.
DIMITRI, M. J. El nuevo libro del árbol: especies forestales de la Argentina oriental. 3. ed. Buenos Aires: El Ateneo, 2000. 124 p. t. 2.

EBY - Entidad Binacional Yacyretá. Estudio hidrológico topográfico y geotécnico del área de Posadas Garupá y Encarnación. Informe Preliminar de Diagnóstico. 1998.

FORGET, P. M. Regeneration ecology of Eperua grandiflora (Caesalpiniaceae), a large-seeded tree in French Guiana. Biotropica, Oxford, v. 24, n. 2, p. 146-156, 1992.

GARELlO, A. Misiones y su Naturaleza. Ley $n^{0}$ 2932- Áreas naturales protegidas - Decreto $n^{\circ}$ 944. Ministerio de Ecología y Recursos Naturales Renovables. 1998.

IGM - Instituto Geográfico Militar. Cartas Topográficas del Instituto Geográfico Militar Argentino. Escala 1:50000. 1938.

KÖPPEN, W. Las zonas del clima. In: Climatología. México: Fondo de Cultura Económica, 1948. 63 p. LORENZI, H. Árvores brasileiras: manual de cultivo de plantas arbóreas nativas do Brasil. Nova Odessa: Plantarum, 1998. v. 1.

MARTINEZ CROVETTO, R. Fitogeografía de Misiones. Bonplandia, Corrientes, v.1, n. 3, 171223, 1963.

MARTINEZ-RAMOS, M. Claros, ciclos vitales de los arboles tropicales y regeneración natural de selvas altas perennifolias. In: GOMES-POMPA, A.; DEL AMO, S. (Eds.). Investigaciones sobre la regeneración de selvas altas en Veracruz, México. México: DF Alhambra, 1985. p. 191-240.

MARTINEZ-RAMOS, M.; ALVAREZ-BUYLLA, E. R. Ecología de poblaciones de plantas en una selva húmeda de México. Boletín de la Sociedad de Botánica de México, México, v. 56, p. 121-153, 1995.

MARTINS, S. S. et al. Efeito da exploração florestal seletiva em uma floresta estacional semidecidual. Revista Árvore, Viçosa, v. 27, n.1, p. 65-70, jan./ fev. 2003.

MORISITA, M. Measuring of the dispersion of individuals and analysis of the distributional patterns. Mem. Fac. Science, Kyushu, v. 2, n. 4, p. 215-235, 1959.

ODUM, E. P. Fundamentos de ecologia. 4. ed. Lisboa: Fundação Colouste Gulbenkian, 1972. 927 p.

REITZ, R.; KLEIN, R. M.; REIS, A. Projeto madeira do Rio Grande do Sul. Porto Alegre: Sudesul, 1988. 525 p.

VAN GROENENDAEL, J. M.; BULLOCK, S. H.; 
PÉREZ-JIMÉNEZ, L. A. Aspects of the population biology of the gregarious tree Cordia elaegnoides in mexican tropical deciduous forest. Journal of Tropical Ecology, Cambridge, v. 12, n.1, p.11-24, Jan. 1996.

VIANA, V. M.; TABANEZ, A. J. A.; MARTINEZ, J .L. A. Restauração e manejo de fragmentos florestais. In: CONGRESSO NACIONAL SOBRE
ESSÊNCIAS NATIVAS, 2., 1992, São Paulo. Anais ... São Paulo: Instituto Florestal, 1992. v. 4, p. 400406.

WEBB, L. S.; TRACEY, J. G.; WILLINS, W. T. Regeneration and pattern in the subtropical rain forest. Journal of Ecology, London, v. 60, n.3, p. 675-695, Nov. 1972. 